\title{
Evaluation of mandibular cortical bone ratio on computed tomography images in patients taking bisphosphonates
}

\author{
Chul-Hong Koo and Jae-Hoon Lee* (D)
}

\begin{abstract}
Background: Bisphosphonate (BP) has the ability to thicken the cortical bone. In addition, it has been reported that the cortical bone thickened by BP has relation to the medication-related osteonecrosis of the jaw (MRONJ). Therefore, the objective of this article is to analyze the ratio as well as thickness of cortical bone in the mandible using computed tomography (CT) and to evaluate it as the predictive factor of MRONJ.

Methods: The thickness of the cortical bone was measured on a paraxial view of the CT showing the mental foramen in 95 patients: 33 patients with MRONJ (3 males, 30 females), 30 patients taking BP without MRONJ (2 males, 28 females), and 32 controls ( 9 males, 28 females). Also, the ratios of the cortical bone to the total bone were obtained using the measured values. Based on these results, we compared the difference of mandibular cortical bone ratio between the three groups.

Results: The average cortical bone thickness was measured as $3.81 \mathrm{~mm}$ in patients with MRONJ, $3.39 \mathrm{~mm}$ in patients taking $\mathrm{BP}$ without MRONJ, and $3.23 \mathrm{~mm}$ in controls. There was only a significant difference between patients with MRONJ and controls $(P<0.05)$. On the other hand, the average mandibular cortical bone ratio was measured as $37.9 \%$ in patients with MRONJ, $27.9 \%$ in patients taking BP without MRONJ, and $23.3 \%$ in controls. There was a significant difference between all groups $(P<0.05)$.

Conclusion: The mandibular cortical bone ratio is large in order of patients with MRONJ, patients taking BP without MRONJ, and controls. This result suggests that the mandibular cortical bone ratio would be very useful to predict the development of MRONJ.
\end{abstract}

Keywords: Bisphosphonate, Medication-related osteonecrosis of the jaw, Mandibular cortical bone ratio

\section{Background}

Bisphosphonate (BP) is widely used in osteoporosis patients [1]. BP is strongly attached to bone matrix and has the function of inhibiting the action of osteoclast [2]. As a result, BP reduces osteoclast action and induces osteoclast apoptosis, thereby reducing bone remodeling. This takes a long time to complete secondary mineralization in existing bone matrix [3-5]. If secondary mineralization persists, the bone matrix density increases and then the cortical bone appears to thicken. This results in increased cortical bone thickness and reduced cancellous bone area [6].

\footnotetext{
* Correspondence: Lee201@dankook.ac.kr

Department of Oral and Maxillofacial Surgery, College of Dentistry, Dankook University, 119 Dandae-ro, Dongnam-gu, Cheonan, South Korea
}

In fact, it was reported that the cortical bone thickness of the femur increased by $1.82 \%$ in patients taking alendronate, one of the BP drugs, while it decreased by $0.31 \%$ in the control group [7]. In addition, the cortical bone of the second metacarpal bone of patients taking BP was thicker than that of patients not taking BP [8]. Similarly, the cortical bone of the mandible in patients taking $\mathrm{BP}$ was thicker than that in the normal subjects [9]. Likewise, several studies have reported that the thickness of the cortical bone is increased when BP is administered [7-10]. According to the leading theory of mechanism of MRONJ development, the capillaries within the bones become embedded in the bones as the secondary mineralization continues. Then, the bone becomes avascular. Because of the injury of the gingiva or invasive surgery, this necrotic bone 
is exposed and healing is not done well. As a result, MRONJ occurs [11]. Recent studies have shown that the thickness of the cortical bone and the incidence of MRONJ are related [12]. In fact, when the thickness of the mandibular cortical bone was measured, the cortical bone of patients with MRONJ was significantly thicker than that of patients taking bisphosphonate without MRONJ and normal subjects [13].

However, the cortical bone thickness itself may be different depending on the individual characteristics [14]. In addition, the thickness of the cortical bone may change according to the intracortical remodeling between the cortical bone and the cancellous bone [15]. Therefore, the aim of this article is to analyze the ratio as well as the thickness of the mandibular cortical bone using the CT and to evaluate it as the predictive factor of MRONJ.

\section{Methods}

\section{Study subjects}

We studied patients who attended the Department of Oral and Maxillofacial Surgery of Dankook University Dental Hospital in South Korea between February 2012 and September 2017. A total of 95 patients were classified into three groups according to the presence of MRONJ or taking BP. The first group was classified into patients with MRONJ (group A), the second group was patients taking $\mathrm{BP}$ without MRONJ (group B), and the last group was patients not taking BP (group C). The following cases were excluded: patients under 65 years of age, with non-menopause, without CT of the entire mandible, with radiotherapy, with osteomyelitis in the mandible, or with oral lesion involving cortical bone in the paraxial view of measurement site. Also, the overall medical history including the age, sex, and the use of BP was examined. In patient taking $\mathrm{BP}$, the administration route and administration period were further investigated. In addition, the MRONJ staging system was also investigated according to the American Association of Oral and Maxillofacial Surgery criteria.

\section{Study methods}

CT

CT radiographs taken with the PHT-60FO (VATECH Corp., Hwa-Sung, Korea) were reconstructed into a paraxial view using Pacsplus viewer 3.2 (Pacsplus, Orange, CA, USA).

\section{CT analysis}

Both sides of the mental foramen in the paraxial view of the CT image were used for one patient. Next, thickness of cortical bone was measured at three parts in each section (Fig. 1).

For cortical bone thickness analysis:

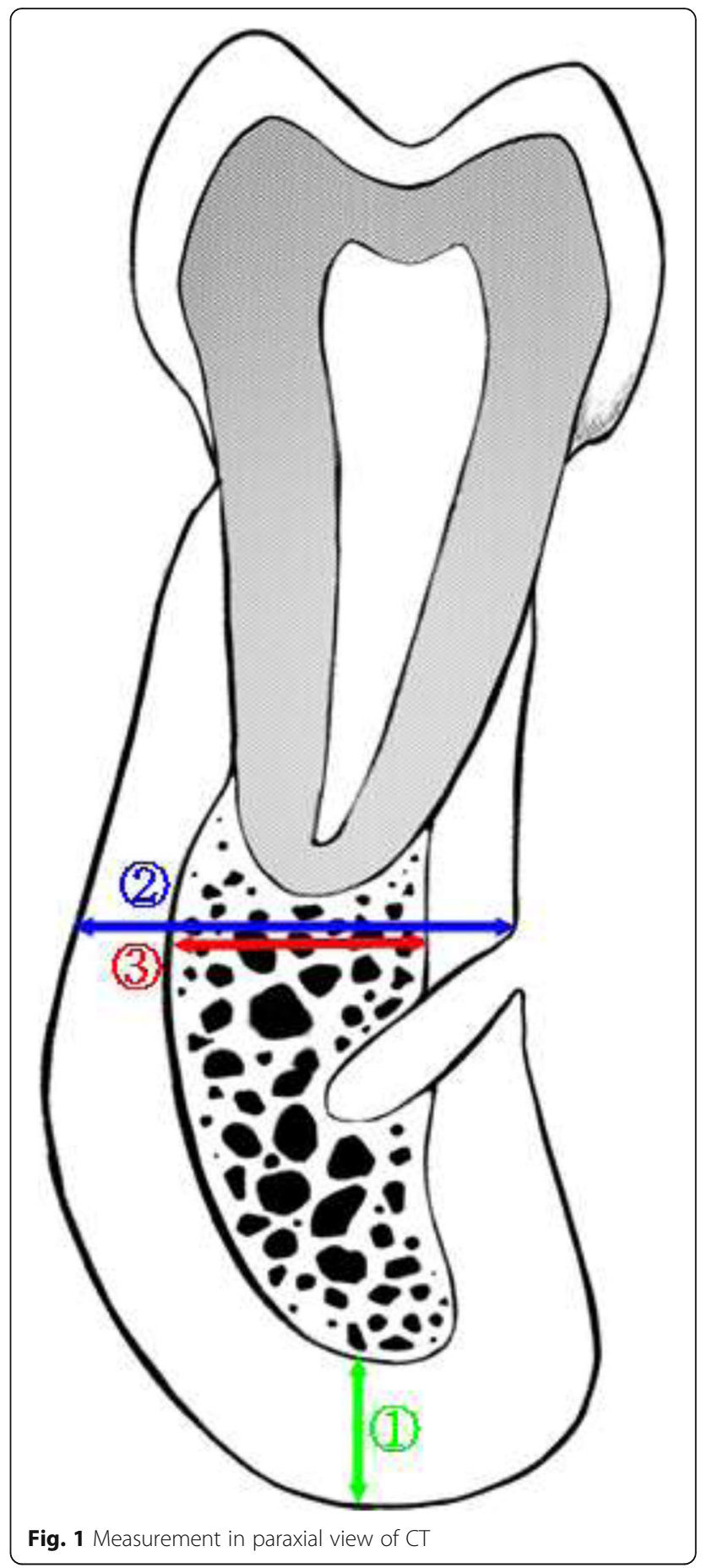

(1) Thickness of the cortical bone in mandibular inferior border

For cortical bone ratio analysis:

(2) Thickness of the total bone on the line parallel to the occlusal plane 
(3) Thickness of the cancellous bone thickness on the line parallel to the occlusal plane

Using the measured values, the mandibular cortical bone ratio was calculated. First, the thickness of the cortical bone is obtained by subtracting the thickness of the cancellous bone from that of the total bone. Then, mandibular cortical bone ratio was calculated as the percentage of thickness of cortical bone to that of total bone by following formula.

$$
\text { Mandibular cortical bone ratio }(\%)=\frac{(2-3)}{(2)} \times 100
$$

\section{Evaluation method}

The mandibular inferior cortical bone thickness was compared among the three groups of patients (those with MRONJ, those taking BP without MRONJ, and controls). Next, mandibular cortical bone ratio was compared among the three groups. In addition, correlation between cortical bone ratio and age was confirmed in each group. Lastly, mandibular cortical bone ratio was compared according to the administration period of $\mathrm{BP}$ in two groups (those with MRONJ and those taking BP without MRONJ).

\section{Statistical analysis}

For comparative analyses of parametric variables in the three groups, one-way analysis of variance (ANOVA) was performed, followed by the Tukey test to validate the results. For comparative analyses of non-parametric variables in the three groups, Kruskal-Wallis test was performed, followed by the Mann-Whitney test to validate the results. In order to examine the correlation between the two variables, Pearson's correlation coefficient was obtained. When the variables did not follow a standard distribution, the mean of the two groups divided by administration period was compared using the Mann-Whitney test. IBM SPSS 21.0 software (IBM Co., Armonk. NY. USA) was used to perform the statistical analyses. Differences were considered statistically significant if the confidence interval $P$ value was less than 0.05 .

\section{Result}

CT images of the patients in the three groups (those with MRONJ, those taking bisphosphonate without MRONJ, and controls) were compared and analyzed to obtain the following results.

\section{Patient groups}

Of the total 95 patients, 81 were women and 14 were men. The mean age was $74.5 \pm 5.6$ years (Table 1 ). Group A had a mean age of $74.5 \pm 6.0$ years, with 30 women and 3 men among 33 patients. Of these, 15 had a history of BP taking more than 4 years and 10 had less than 4 years. Eight could not remember accurately how long they took BP. In addition, 8 had DM, and 25 did not have DM. According to MRONJ staging, stage 1 was 1 , stage 2 was 27, and stage 3 was 5 .

Group B had a mean age of $74.3 \pm 5.8$ years, with 28 women and 2 men among 30 patients. Of these, 12 had more than 4 years of BP taking, and 12 had less than 4 years. Six could not remember accurately how long they took BP. There were 4 people with DM and 24 people without DM.

Group $C$ had a mean age of $74.8 \pm 5.1$ years, with 23 women and 9 men among 32 patients. There were 8 people with DM and 24 people without DM.

\section{Comparison of mandibular inferior cortical bone thickness}

The average cortical bone thickness was measured as $3.81 \mathrm{~mm}$ in group A, $3.39 \mathrm{~mm}$ in group B, and $3.23 \mathrm{~mm}$ in group $C$. There was a significant difference between group $\mathrm{A}$ and group $\mathrm{C}$ when comparing each cortical bone thickness $(P<0.05)$. On the other hand, there was no significant difference between group A and group $B$ and between group $\mathrm{B}$ and group $\mathrm{C}$ (Table 2).

\section{Comparison of mandibular cortical bone ratio}

The average mandibular cortical bone ratio was measured at the mental foramen, which was $37.9 \%$ in group A, $27.9 \%$ in group B, and $23.3 \%$ in group C. There was a significant difference between all groups when each cortical bone ratio was compared $(P<0.05)$, (Table 3$)$.

\section{Correlation between cortical bone ratio and diabetes mellitus}

The cortical bone ratio between the groups was compared according to the presence or absence of diabetes mellitus (DM). In the groups with DM, there was a significant difference between group $\mathrm{A}$ and group $\mathrm{C}$ and between group $\mathrm{B}$ and group $\mathrm{C}(P<0.05)$. But there was no significant difference between group A and group B (Table 4). On the other hand, there was a significant difference between all groups without DM $(P<0.05)$ (Table 5$)$.

\section{Correlation between cortical bone ratio and age}

As a result of analyzing the correlation cortical bone ratio and age, there was no significant correlation between the cortical bone ratio and age in each group (Table 6).

Comparison of cortical bone ratio by administration period Patients who did not know accurately their administration period were excluded in this comparison. As a result of analyzing the cortical bone ratio by 
Table 1 Gender, age, administration period, DM, and MRONJ staging of each group

\begin{tabular}{|c|c|c|c|c|}
\hline & & $\begin{array}{l}\text { Group A } \\
(n=33)\end{array}$ & $\begin{array}{l}\text { Group B } \\
(n=30)\end{array}$ & $\begin{array}{l}\text { Group C } \\
(n=32)\end{array}$ \\
\hline \multirow[t]{2}{*}{ Gender } & Male & 3 & 2 & 9 \\
\hline & Female & 30 & 28 & 23 \\
\hline \multirow[t]{2}{*}{ DM } & Presence & 8 & 4 & 8 \\
\hline & Absence & 25 & 26 & 24 \\
\hline \multirow[t]{4}{*}{ Age } & Years (mean \pm SD) & $74.5 \pm 6.0$ & $74.3 \pm 5.8$ & $74.8 \pm 5.1$ \\
\hline & $65 \leq$ years $<75(n)$ & 21 & 15 & 17 \\
\hline & $75 \leq$ years $<85(n)$ & 11 & 14 & 15 \\
\hline & $85 \leq$ years $(n)$ & 1 & 1 & 0 \\
\hline \multirow[t]{3}{*}{ Administration period $(n=49)$} & Years (mean \pm SD) & $6.3 \pm 5.3$ & $4.8 \pm 4.4$ & - \\
\hline & $<4$ years $(n)$ & 10 & 12 & - \\
\hline & $\geq 4$ years $(n)$ & 15 & 12 & - \\
\hline \multirow[t]{3}{*}{ MRONJ Staging } & Stage 1 & 1 & - & - \\
\hline & Stage 2 & 27 & - & - \\
\hline & Stage 3 & 5 & - & - \\
\hline
\end{tabular}

A: patients with MRONJ

B: patients taking BP without MRONJ

C: controls

administration period, there was no significant difference in each group (Table 7).

\section{Discussion}

Several studies have observed radiologic features of patients taking BP. It has been reported that the use of bone scan can detect symptom-free osteonecrosis in patients treated with BP and the extent and characteristics of osteolytic lesions can be determined using CT and MRI [16]. Rocha et al. [17] maintained that the change of bone was early confirmed by panoramic radiographs making it easy to diagnose MRONJ. In many kinds of radiological examinations including panoramic radiographs, periapical radiographs, CT, and MRI, it was observed that osteolysis, bone deposition, thickening of lamina dura, and cortical margins when taking BP [18]. In addition, it was reported that patients taking BP showed cortical bone erosion, sequestrum formation, or pathological fracture of the jaw in panoramic radiographs [19].

Table 2 Mandibular cortical bone thickness in each group

\begin{tabular}{lllllll}
\hline & Group & Mean & SD & \multicolumn{3}{l}{ Significance } \\
\cline { 5 - 7 } & & & & A-B & A-C & B-C \\
\hline Cortical bone & A & 3.81 & \pm 0.89 & 0.211 & $0.028^{*}$ & 0.863 \\
thickness (mm) & B & 3.39 & \pm 0.96 & & & \\
& C & 3.23 & \pm 0.86 & & & \\
& & & & & &
\end{tabular}

A: patients with MRONJ

B: patients taking BP without MRONJ

C: controls

*Significant at the level of $P<0.05$
Moreover, there have been studies quantitatively evaluating changes of the mandible associated with $\mathrm{BP}$ on radiographs. Torres et al. [12, 13] compared the thickness, cross-sectional area, and volume of the mandibular cortical bone with MRONJ patients and normal subjects who did not take BP. As a result, MRONJ patients showed larger values than normal subjects. Also, they compared the thickness of the mandibular inferior cortical bone in panoramic radiographs among patients with MRONJ, patients taking bisphosphonate without MRONJ, and normal subjects. The thickness of the cortical bone was significantly thicker in patients with MRONJ $(6.81 \pm 1.35 \mathrm{~mm})$ than those without MRONJ $(5.44 \pm 1.09 \mathrm{~mm})$ and normal subjects $(4.79 \pm 0.85 \mathrm{~mm})$. According to Hamada et al. [20], it was observed that MRONJ patients had thicker cortical bone and higher radiodensity of cancellous bone than normal subjects on CT images. In addition, Iwata et al. [21] measured the thickness of the mandibular buccal and lingual cortical bone in three groups: patients with MRONJ, patients

Table 3 Mandibular cortical bone ratio in each group

\begin{tabular}{llllllll}
\hline & Group & Mean & SD & \multicolumn{3}{l}{ Significance } \\
\cline { 5 - 7 } & & & & A-B & A-C & B-C \\
\hline Cortical bone ratio (\%) & A & 37.9 & \pm 7.1 & $0.000^{*}$ & $0.000^{*}$ & $0.006^{*}$ \\
& B & 27.9 & \pm 5.4 & & & \\
& C & 23.3 & \pm 4.1 & & & \\
\hline
\end{tabular}

A: patients with MRONJ

B: patients taking BP without MRONJ

C: controls

* Significant at the level of $P<0.05$ 
Table 4 Mandibular cortical bone ratio in each group with DM

\begin{tabular}{lllllll}
\hline & Group & Mean & SD & \multicolumn{4}{c}{ Significance } \\
\cline { 5 - 7 } & & & & A-B & A-C & B-C \\
\hline Cortical bone ratio (\%) & A & 36.0 & \pm 6.8 & 0.683 & $0.002^{*}$ & $0.016^{*}$ \\
& B & 33.5 & \pm 3.6 & & & \\
& C & 24.1 & \pm 4.7 & & & \\
\hline
\end{tabular}

A: patients with MRONJ

B: patients taking BP without MRONJ

C: controls

*Significant at the level of $P<0.05$

taking osteoporosis medication without MRONJ, and non-osteoporotic group. As a result, they found that the cortical bone of patients with MRONJ was significantly thicker than that of the other groups. But they could not find significant difference between patients taking osteoporosis medicine without MRONJ and normal subjects. On the other hand, according to the measurement of the mandibular cortical bone thickness in our study, there was a significant difference only between MRONJ group and normal group. In other words, it is difficult to distinguish patients taking $\mathrm{BP}$ without MRONJ from other groups by cortical bone thickness. Therefore, it is difficult to predict the incidence of MRONJ using only cortical bone thickness on patients taking BP.

It was reported that aging of bone tissue and reduction of bone density begins from 45 years of age and bone loss of the cortical bone occurs mainly from about 65 years old [14]. Also, the cortical bone thickness itself varies depending on individual characteristics, such as the shape of the face, occlusal force, and age [22]. Thus, the size of the jaw and the thickness of the cortical bone can vary from person to person. In addition, Zebaze et al. [15] found that cortical bone thickness can be changed by intracortical remodeling between the cortical bone and cancellous bone. Therefore, we examined not only merely the thickness of the cortical bone but also the ratio of the cortical bone to total bone in this study. The mandibular cortical bone ratio was measured in patients with MRONJ, patients taking BP without MRONJ, and controls, and the difference between these groups was confirmed. As a result, mandibular cortical bone ratio was significantly larger in patients with MRONJ than

Table 5 Mandibular cortical bone ratio in each group without DM

\begin{tabular}{lllllll}
\hline & Group & Mean & SD & \multicolumn{4}{c}{ Significance } \\
\cline { 5 - 7 } & & & & A-B & A-C & B-C \\
\hline Cortical bone ratio (\%) & A & 38.5 & \pm 7.3 & $0.000^{*}$ & $0.000^{*}$ & $0.006^{*}$ \\
& B & 27.0 & \pm 5.2 & & & \\
& C & 23.0 & \pm 3.9 & & & \\
\hline
\end{tabular}

A: patients with MRONJ

B: patients taking BP without MRONJ

C: controls

*Significant at the level of $P<0.05$
Table 6 Correlation between mandibular cortical bone ratio and age in each group

\begin{tabular}{lll}
\hline Group & Pearson & \\
\cline { 2 - 3 } & Correlation coefficient & Significance \\
\hline A & -0.294 & 0.097 \\
B & -0.337 & 0.069 \\
C & -0.247 & 0.174 \\
\hline A: patients with MRONJ & \\
B: patients taking BP without MRONJ & \\
C: controls & \\
*Significant at the level of $P<0.05$ &
\end{tabular}

in patients taking BP without MRONJ and controls. It was also found that the mandibular cortical bone ratio was significantly higher in the patients taking BP without MRONJ than in the controls, that is, the measurement of the mandibular cortical bone ratio showed a significant difference between all groups.

DM can cause bone quality deterioration through microvascular ischemia, endothelial dysfunction, reduced bone remodeling, osteoblast, and osteoclast apoptosis and induce changes in immune cell function and inflammation. Therefore, DM increases the risk of chronic infection and MRONJ [23]. In this study, mandibular cortical bone ratios were compared according to the presence or absence of DM. When cortical bone ratio was examined in patients with DM in each group, there was no significant difference between the patients with MRONJ and the patients taking BP without MRONJ (Table 4). On the other hand, in patients without DM, there was a significant difference between the patients with MRONJ and the patients taking BP without MRONJ (Table 5). In other words, in patients with $\mathrm{DM}$, cortical bone ratio of the group with MRONJ is similar to that of the group taking BP without MRONJ. These results suggest that patients taking $\mathrm{BP}$ without MRONJ are more likely to develop MRONJ in the presence of DM.

Additionally, the correlation between the mandibular cortical bone ratio and age was analyzed. As a result, the ratio decreased with increasing age in all groups, but it did not show any significant correlation. This is because

Table 7 Comparison of cortical bone ratio by administration period in A and B group

\begin{tabular}{llllc}
\hline Group & Administration period & Mean & SD & Significance \\
\hline A & $<4$ years & 37.9 & 7.0 & 0.807 \\
& $\geq 4$ years & 36.6 & 6.7 & \\
B & $<4$ years & 26.6 & 4.5 & 0.630 \\
& $\geq 4$ years & 28.8 & 5.9 & \\
\hline
\end{tabular}

A: patients with MRONJ

B: patients taking BP without MRONJ

*Significant at the level of $P<0.05$ 
the subjects were confined to 65 years or older, not all ages.

The two groups taking BP (those with MRONJ and those taking $\mathrm{BP}$ without MRONJ) were subdivided by administration period (the 4 years standard). The reason why they are divided by administration period based on 4 years standard is that the prevalence of MRONJ in patients taking BP treatment for more than 4 years increased significantly to $0.21 \%$ [2]. Then, difference of cortical bone ratio by administration period was analyzed. It was resulted that there was no significant difference between patients with MRONJ and patients taking BP without MRONJ. This may be due to the fact that the number of patients who were precisely examined for the administration period in each group was low, and the administration period was not accurate because it depends on the patient's statements. In addition, the difference of the administration route would have affected the outcome.

In this study, the cortical bone thickness and ratio were measured near the mental foramen, which was measured from the basal bone, not the alveolar bone where the MRONJ is predominant. There are two reasons why reference point was near the mental foramen. First, most MRONJ patients have the alveolar bone that has already invaded cortical bone destruction or has undergone bone resorption due to poor periodontal status. Accordingly, it is difficult to set a reference point for thickness and ratio measurement. Lastly, when osteoporosis medication is taken, it acts systemically and it is difficult to occur specific cortical bone thickness changes only in specific areas of the mandible.

Previous studies have measured cortical bone thickness in relation to MRONJ, but there was no significant difference between patients taking BP without MRONJ and normal subjects. However, in this study, we could also find significant differences between patients taking $\mathrm{BP}$ without MRONJ and normal subjects by using mandibular cortical bone ratio. Therefore, if further research is progressed in the future, it may be used as a predictor of the incidence of MRONJ in patients who must have oral surgery including extraction.

\section{Conclusion}

We compared the CT findings of patients with MRONJ, patients taking BP without MRONJ, and controls, and the following results were obtained. In measurement of cortical bone thickness, that of patients with MRONJ (group A) is significantly thicker than that of the controls (group C). However, there is no significant difference between patients with MRONJ (group A) and patients taking BP without MRONJ (group B). And, there is no significant difference between patients taking
BP without MRONJ (group B) and controls (group C). On the other hand, in measurement of cortical bone ratio, patients with MRONJ (group A), patients taking BP without MRONJ (group B), and controls (group C) are large in order, and there is a significant difference between all groups.

To conclude, it is considered that "ratio of cortical bone to total bone in mandible" would be one of the good predictive factors for MRONJ in the future.

\section{Abbreviations}

BP: Bisphosphonate; CT: Computed tomography; MRONJ: Medication-related osteonecrosis of the jaw

\section{Funding}

This study was supported by the research fund of Dankook University in 2016.

Availability of data and materials

Please contact the author for data requests.

\section{Authors' contributions}

CHK participated in the design of the study, collection, analysis, and interpretation of the data, and is involved in the drafting the manuscript. JHL conceived the study, participated in its design and coordination, and helped to draft the manuscript. Both authors read and approved the final manuscript.

Ethics approval and consent to participate

Not applicable.

\section{Consent for publication}

Not applicable.

\section{Competing interests}

The authors declare that they have no competing interests.

\section{Publisher's Note}

Springer Nature remains neutral with regard to jurisdictional claims in published maps and institutional affiliations.

Received: 30 April 2018 Accepted: 8 June 2018

Published online: 05 July 2018

\section{References}

1. Chen JS, Sambrook PN (2011) Antiresorptive therapies for osteoporosis: a clinical overview. Nat Rev Endocrinol 8(2):81-91

2. Russell RG, Watts NB, Ebetino FH, Rogersm MJ (2008) Mechanisms of action of bisphosphonates: similarities and differences and their potential influence on clinical efficacy. Osteoporos Int 19(6):733-759

3. Benford HL, McGowan NW, Helfrich MH, Nuttall ME, Rogers MJ (2001) Visualization of bisphosphonate-induced caspase-3 activity in apoptotic osteoclasts in vitro. Bone 28(5):465-473

4. Plotkin LI, Weinstein RS, Parfitt AM, Roberson PK, Manolagas SC, Bellido T (1999) Prevention of osteocyte and osteoblast apoptosis by bisphosphonates and calcitonin. J Clin Invest 104(10):1363-1374

5. Stepan JJ, Alenfeld F, Boivin G, Feyen JH, Lakatos P (2003) Mechanisms of action of antiresorptive therapies of postmenopausal osteoporosis. Endocr Regul 37(4):225-238

6. Seeman $\mathrm{E}$ (2010) Bone morphology in response to alendronate as seen by high-resolution computed tomography: through a glass darkly. J Bone Miner Res 25(12):2553-2557

7. Beck TJ, Lewiecki EM, Miller PD, Felsenberg D, Liu Y, Ding B et al (2008) Effects of denosumab on the geometry of the proximal femur in postmenopausal women in comparison with alendronate. J Clin Densitom 11(3):351-359

8. Hyldstrup L, Jorgensen JT, Sorensen TK, Baeksgaard L (2001) Response of cortical bone to antiresorptive treatment. Calcif Tissue Int 68(3):135-139

9. Taniguchi T, Ariji Y, Nozawa M, Naitoh M, Kuroiwa Y, Kurita K et al (2016) Computed tomographic assessment of early changes of the mandible in 
bisphosphonate-treated patients. Oral Surg Oral Med Oral Pathol Oral Radiol 122(3):362-372

10. Bravenboer N, Papapoulos SE, Holzmann P, Hamdy NA, Netelenbos J, Lips P (1999) Bone histomorphometric evaluation of pamidronate treatment in clinically manifest osteoporosis. Osteoporos Int 9(6):489-493

11. Marx RE, Sawatari Y, Fortin M, Broumand V (2005) Bisphosphonate-induced exposed bone (osteonecrosis/osteopetrosis) of the jaws: risk factors, recognition, prevention, and treatment. J Oral Maxillofac Surg 63(11):1567-1575

12. Torres SR, Chen CS, Leroux BG, Lee PP, Hollender LG, Santos EC et al (2012) Mandibular cortical bone evaluation on cone beam computed tomography images of patients with bisphosphonate-related osteonecrosis of the jaw. Oral Surg Oral Med Oral Pathol Oral Radiol 113(5):695-703

13. Torres SR, Chen CS, Leroux BG, Lee PP, Hollender LG, Lloid M et al (2015) Mandibular inferior cortical bone thickness on panoramic radiographs in patients using bisphosphonates. Oral Surg Oral Med Oral Pathol Oral Radiol 119(5):584-592

14. Helfen T, Sprecher CM, Eberli U, Gueorguiev B, Muller PE, Richards RG et al (2017) High-resolution tomography-based quantification of cortical porosity and cortical thickness at the surgical neck of the humerus during aging. Calcif Tissue Int 101(3):271-279

15. Zebaze R, Seeman E (2015) Cortical bone: a challenging geography. J Bone Miner Res 30(1):24-29

16. Chiandussi S, Biasotto M, Dore F, Cavalli F, Cova MA, Di Lenarda R (2006) Clinical and diagnostic imaging of bisphosphonate-associated osteonecrosis of the jaws. Dentomaxillofac Radiol 35(4):236-243

17. Rocha GC, Jaguar GC, Moreira CR, Neves EG, Fonseca FP, Pedreira EN (2012) Radiographic evaluation of maxillofacial region in oncology patients treated with bisphosphonates. Oral Surg Oral Med Oral Pathol Oral Radiol 114(5 Suppl):S19-S25

18. Arce K, Assael LA, Weissman JL, Markiewicz MR (2009) Imaging findings in bisphosphonate-related osteonecrosis of jaws. J Oral Maxillofac Surg 67(5 Suppl):75-84

19. Cardoso CL, Barros CA, Curra C, Fernandes LM, Franzolin SO, Junior JS et al (2017) Radiographic findings in patients with medication-related osteonecrosis of the jaw. Int J Dent 3190301. https://doi.org/10.1155/2017/3190301.

20. Hamada H, Matsuo A, Koizumi T, Satomi T, Chikazu D (2014) A simple evaluation method for early detection of bisphosphonate-related osteonecrosis of the mandible using computed tomography. J Craniomaxillofac Surg 42(6):924-929

21. Iwata E, Akashi M, Kishimoto M, Kusumoto J, Hasegawa T, Furudoi S et al (2017) Meaning and limitation of cortical bone width measurement with DentaScan in medication-related osteonecrosis of the jaws. Kobe J Med Sci 62(5):114-119

22. Swasty D, Lee JS, Huang JC, Maki K, Gansky SA, Hatcher D et al (2009) Anthropometric analysis of the human mandibular cortical bone as assessed by cone-beam computed tomography. J Oral Maxillofac Surg 67(3):491-500

23. Peer A, Khamaisi M (2015) Diabetes as a risk factor for medication-related osteonecrosis of the jaw. J Dent Res 94(2):252-260

\section{Submit your manuscript to a SpringerOpen ${ }^{\circ}$ journal and benefit from:}

- Convenient online submission

- Rigorous peer review

Open access: articles freely available online

- High visibility within the field

Retaining the copyright to your article

Submit your next manuscript at $>$ springeropen.com 